\title{
Outpatient chemotherapy with gemcitabine and oxaliplatin in patients with biliary tract cancer
}

\author{
J Harder ${ }^{*, 1,4}$, B Riecken ',4, O Kummer', C Lohrmann², F Otto ${ }^{3}$, H Usadel', M Geissler', O Opitz' and H Henß $\beta^{3}$ \\ 'Department of Gastroenterology and Hepatology, Freiburg University Hospital, Hugstetterstr. 55, Freiburg D-79106, Germany; ${ }^{2}$ Department of \\ Radiology, Freiburg University Hospital, Freiburg, Germany; ${ }^{3}$ Department of Hematology and Oncology, Freiburg, Germany
}

This phase II study was conducted to determine the efficacy and toxicity of a gemcitabine (GEM) and oxaliplatin (OX) chemotherapy protocol in patients with unresectable biliary tract cancer (BTC). Patients were treated with GEM I000 mg m ${ }^{-2}$ (30 min infusion) on days I, 8, I5, and OX $100 \mathrm{mg} \mathrm{m}^{-2}$ (2 h infusion) on days I and I5 (gemcitabine and oxaliplatin (GEMOX-3 protocol), repeated every 28 days. The data were collected according to the Simon 2 -stage design for a single centre phase II study $(\alpha=0.05 ; \beta=0.2)$. Primary end point was response rate; secondary end points were time-to-progression (TTP), median survival, and safety profile. Thirty-one patients were enrolled in the study between July 2002 and April 2005. Therapeutic responses were as follows: partial response in eight patients $(26 \%, 95 \%$ confidence interval $(\mathrm{Cl}) 14-44)$, stable disease in 14 patients $(45 \%, 95 \% \mathrm{Cl} 29-62)$, resulting in a disease control rate of $71 \%$. Nine patients $(29 \%, 95 \% \mathrm{Cl} 16-47)$ had progressive disease. Median TTP was 6.5 months. Median overall survival was II months. Common Toxicity Criteria (CTC) Grade 3-4 toxicities were transient thrombocytopenia (23\%), peripheral sensory neuropathy (19\%), leucopenia (16\%), and anaemia (10\%). In conclusion the GEMOX-3 protocol is active and well tolerated in patients with advanced BTC. It can be applied in an outpatient setting with three visits per month only.

British Journal of Cancer (2006) 95, 848-852. doi:I0.1038/sj.bjc.6603334 www.bjcancer.com

Published online 12 September 2006

(c) 2006 Cancer Research UK

Keywords: biliary tract neoplasms; chemotherapy; gemcitabine; oxaliplatin

Biliary tract cancer (BTC) is a heterogeneous tumour entity with a dismal prognosis. Incidence and mortality rates of intrahepatic cholangiocarcinomas are rising, whereas gall bladder and extrahepatic cholangiocarcinoma incidences are slightly declining (Patel, 2001, 2002; Khan et al, 2002). At present, surgery is the only curative treatment option for BTC. However, less than $25 \%$ of patients are resectable at presentation with high relapse rates after surgery (Oertli et al, 1993; de Groen et al, 1999). The 5-year survival rates after resection for intrahepatic cholangiocarcinomas vary from $8-47 \%$ (Nakeeb et al, 2002).

A benefit over no therapy or best supportive care has been demonstrated for palliative treatment options with either surgical or endoscopic biliary drainage, and/or chemotherapy (Farley et al, 1995; Chang et al, 1998). However, no standard chemotherapy exists, owing to the rarity, and heterogeneity of the disease.

In recent phase-II trials, gemcitabine (GEM), a pyrimidine analogue, has been shown to be an active single agent therapy in BTC. For this drug objective response rates (RR) in a range from 8 to $60 \%$ have been reported (Raderer et al, 1999; Valencak et al, 1999; Gallardo et al, 2001; Gebbia et al, 2001; Kubicka et al, 2001; Penz et al, 2001). In addition, various combination therapies have been investigated in the setting of phase II studies with RRs between 9.5 and 53\% (Gebbia et al, 2001; Patt et al, 2001; Doval

\footnotetext{
*Correspondence: Dr J Harder;
}

E-mail: harder@medizin.ukl.uni-freiburg.de

${ }^{4}$ These authors contributed equally to this work.

Received 8 May 2006; revised 27 July 2006; accepted 27 July 2006; published online 12 September 2006 et al, 2002; Kuhn et al, 2002; Nehls et al, 2002; Taieb et al, 2002; Kornek et al, 2004; Patt et al, 2004; Alberts et al, 2005; Ducreux et al, 2005). Overall, most favourable RRs in BTC were reached with protocols that combine cisplatin (CDDP) with 5-fluorouracil or GEM (Doval et al, 2002; Taieb et al, 2002; Ducreux et al, 2005).

Another combination regimen, which replaces CDDP by the third generation platinum analogue oxaliplatin (OX) could help to reduce the emetic and potential renal toxicity of CDDP without loss of treatment efficacy (Extra et al, 1998). A French study used the combination of GEM and OX in pancreatic cancer with promising results. Because of some similarities in tumour biology and response to chemotherapeutic agents between pancreatic and BTC we reasoned that gemcitabine and oxaliplatin (GEMOX) might be active in BTC as well. Therefore, the rationale for our study was to design a patient friendly protocol, with two in other cancers well tolerated and active substances. In the study by Louvet et al (2002) the two substances were given on 2 consecutive days with relatively long infusion times (fixed dose rate) for GEM. In contrast, we chose to apply GEM as an infusion over $30 \mathrm{~min}$ followed by $\mathrm{OX}$ as a $2 \mathrm{~h}$ infusion on the same day in an outpatient setting.

\section{PATIENTS AND METHODS}

\section{Patients}

Patients included in the study were required to meet the following criteria: 18-75 years old, written informed consent, histologically 
or cytologically confirmed and non-resectable or metastatic BTC, one or more bidimensionally measurable lesions on computed tomography (CT) or magnetic resonance imaging (MRI), Karnofsky Score $\geqslant 70 \%$, leucocyte $\geqslant 3 \times 10^{9} 1^{-1}$, platelets $\geqslant 100 \times 10^{9} 1^{-1}$, serum creatinine $<2 \mathrm{mg} \mathrm{dl}^{-1}$, and bilirubin $<3 \mathrm{mg} \mathrm{dl}^{-1}$, a life expectancy over 3 months, absence of cholangitis or carcinoma of the ampulla of Vater. Primary gastrointestinal cancers other than BTC were excluded by upper endoscopy, colonoscopy, and multislice CT scan. Women were either postmenopausal or were using adequate contraception with a negative pregnancy test at study entry. Patients were excluded in case of preexisting peripheral neuropathy National Cancer Institute-Common Toxicity Criteria (NCI CTC) $>$ grade 1 , use of major surgery or chemotherapy within 1 month, or radiotherapy within 12 months of study entry. Patients were allowed to have received prior chemotherapy (not including GEM or OX) for advanced disease or in the adjuvant setting. The baseline characteristics at study entry are given in Table 1. Patients with severe cardiovascular or pulmonary disease (NYHA III-IV, global respiratory insufficiency), symptomatic cerebral metastases, incompatibility with or allergy to platinol derivates, pregnancy, or lactation, serious infection, additional malignancies other than completely excised in situ carcinoma of the cervix or non-melanomatous skin cancer, and current alcohol or drug addiction were also excluded from the study. The study was carried out according to the Declaration of Helsinki Principles, the Good Clinical Practice criteria and was approved by the local ethics committee. The study was performed without sponsoring.

\section{Treatment plan}

All patients received the treatment protocol GEMOX-3 on an outpatient basis as follows: first GEM $1000 \mathrm{mg} \mathrm{m}^{-2}$ as infusion over $30 \mathrm{~min}$ on days 1,8 , and 15 , thereafter OX $100 \mathrm{mg} \mathrm{m}^{-2}$ over $2 \mathrm{~h}$ on days 1 and 15. Treatment was repeated every 28 days. NCI CTC version 2.0 was used for toxicity assessment. In addition a separate neurotoxicity scale for the assesment of OX-induced sensory neuropathy was used (Extra et al, 1998). Clinical examination including measurement of sensory neuropathy, blood count, and liver function tests were performed with each visit. In case of specific cumulative peripheral sensory neuropathy NCI CTC $>$ grade 1 persisting over 7 days, the dose of OX was reduced to $75 \mathrm{mg} \mathrm{m}^{-2}$. Oxaliplatin was stopped in case of grade 3 or 4 peripheral sensory neuropathy. Treatment was subsequently continued according to the same schedule, but with GEM alone. With leucocytes $<3.0 \times 10^{9} 1^{-1}$ or platelet count $<100 \times 10^{9} 1^{-1}$ treatment was interrupted for 7 days. These criteria were applied to any day of treatment administration. If recovery occurred within the 7 days, treatment was continued without dose reduction, otherwise the dosages of GEM and OX were reduced to 75\%. Doses omitted were given within the two subsequent weeks. After treatment discontinuation for more than 3 weeks the patients went off study. Treatment was continued until disease progression, unacceptable toxicity despite dose modification or patient withdrawal.

\section{Treatment evaluation and statistics}

The data were collected according to Simon's optimal two-stage design for a single centre study (Simon, 1989). According to this design, an additional 19 patients were to be enrolled in case of a minimum RR of $10 \%$ in the first 10 patients. Thus, the planned minimum sample size was 29 . In case of more than three responses in the total of 29 patients (RR of at least $10 \%$ ), the regimen was considered active with $\alpha=0.05 ; \beta=0.2$. All enrolled patients were included in the intention-to-treat analysis.

The primary end point was tumour response according the response evaluation criteria in solid tumours guidelines (Therasse
Table I Baseline characteristics of 3 I patients with BTC

\begin{tabular}{|c|c|c|}
\hline Characteristic & $N$ & (\%) \\
\hline \multicolumn{3}{|l|}{ Sex } \\
\hline Male & 13 & 42 \\
\hline Female & 18 & 58 \\
\hline \multicolumn{3}{|l|}{ Age, years } \\
\hline Median & 63 & \\
\hline Range & $38-75$ & \\
\hline \multicolumn{3}{|l|}{ Karnofsky score } \\
\hline 70 & । & 3 \\
\hline 80 & 4 & 13 \\
\hline 90 & 6 & 19 \\
\hline 100 & 20 & 65 \\
\hline \multicolumn{3}{|l|}{ Primary cancer site } \\
\hline Gallbladder & 10 & 32 \\
\hline Intrahepatic bile ducts & 14 & 45 \\
\hline Extrahepatic & 7 & 23 \\
\hline \multicolumn{3}{|l|}{ Disease at presentation } \\
\hline Locally advanced & 3 & 10 \\
\hline Metastatic & 28 & 90 \\
\hline \multicolumn{3}{|l|}{ Metastatic sites } \\
\hline Liver & 14 & 45 \\
\hline Lung & 6 & 19 \\
\hline Lymph nodes & 19 & 61 \\
\hline \multicolumn{3}{|l|}{ Prior therapy } \\
\hline None & 17 & 55 \\
\hline Surgery & 7 & 23 \\
\hline Endoscopic biliary stenting & 6 & 19 \\
\hline Radiochemotherapy and stent & । & 3 \\
\hline Chemotherapy & 0 & 0 \\
\hline \multicolumn{3}{|l|}{ Presenting symptom } \\
\hline Pain & 16 & 52 \\
\hline Jaundice & 5 & 16 \\
\hline Weight loss & 4 & 13 \\
\hline None & 2 & 6 \\
\hline Fever & 2 & 6 \\
\hline Fatigue & 2 & 6 \\
\hline Total & 20 & 65 \\
\hline Endoscopic biliary stenting during chemotherapy & 3 & 10 \\
\hline
\end{tabular}

BTC, biliary tract cancer.

et al, 2000). The response was specified in percent with 95\%-CI calculated by the modified Wald method (Agresti, 1998). Secondary end points were time-to-progression (TTP), overall survival (OS), and toxicity. Time-to-progression was determined from the first day of treatment until tumour progression assessed by CT scan or MRI. OS was determined from first day of treatment until death. Time-to-progression and OS data were analysed by means of the Kaplan-Meier method.

\section{RESULTS}

\section{Patient characteristics}

The median duration of follow up was 13 months. The cutoff date for data analysis was 21 December 2005. Thirty-one consecutive patients at the Department of Medicine of the University of Freiburg, Germany, were enrolled from July 2002 to April 2005. Demographics and other baseline characteristics are summarised in Table 1. The study included 13 men and 18 women with a median age of 63 years $(38-75)$. The primary cancer site has been: 
$14(45 \%)$ intrahepatic bile ducts, $10(32 \%)$ gallbladder, and $7(23 \%)$ extrahepatic bile ducts. The baseline characteristics are similar to other phase II studies reported. Ampullary carcinomas were excluded.

\section{Safety and response}

A total of 140 cycles of chemotherapy (420 treatment days) were delivered during the study. The median number of cycles was 4 (range $0.66-10$ ). Three patients did not complete the first two cycles and were considered as progressive disease. One patient died of duodenal perforation owing to tumour infiltration without having completed the first cycle (received $\mathrm{d} 1$ and $\mathrm{d} 8$ only). In another two patients, chemotherapy was stopped after 1.66 cycles because of malignant biliary obstruction with consecutive development of an intrahepatic abscess and a cholangitis, respectively. Despite the absence of neutropenia, the immediate start of an appropriate antibiotic treatment, and adequate biliary drainage, both patients subsequently died of cholangiosepsis. Deaths in both patients had been attributed to tumour progression, rather than treatment related, although the latter could not completely be ruled out. All 31 patients were assessable for toxicity. Treatment delays of any reason were necessary in 27 out of 31 patients (87\%). In 13 (42\%) patients dose reductions had to be performed. In seven patients $(23 \%)$, the dose of GEM and OX had to be reduced owing to bone marrow toxicity. In another six patients (19\%), OX had to be reduced and later discontinued owing to peripheral sensory neuropathy. Sixty-six percent of treatment delays were caused by bone marrow toxicity, especially thrombocytopenia below $100 \times 10^{9} 1^{-1} \quad(n=69 / 54 \%)$. Another major reason for delay of treatment were infections (9\%), with febrile neutropenia in two patients. Toxicities according to NCI CTC are summarised in Table 2.

Despite these delays and dose reductions, eight patients (26, $95 \%$ CI $14-44 \%)$ had a partial response and $14(45,95 \%$ CI $29-62 \%$ ) had stable disease. Thus, tumour control was achieved in

Table 2 Grade $3-4 \mathrm{NCl} \mathrm{CTC}$ toxicities $(n=31)$ worst toxicity (all cycles) per patient

\begin{tabular}{lcr}
\hline Grade 3-4 toxicity & N & (\%) \\
\hline Leucocytopenia (grade 3) & 5 & 16 \\
Febrile neutropenia & 2 & 7 \\
Thrombocytopenia & 7 & 23 \\
Anaemia (grade 3) & 3 & 10 \\
Vomiting & 0 & 0 \\
Diarrhoea & 0 & 0 \\
Peripheral sensory neuropathy & 6 & 19 \\
Mucositis & 0 & 0 \\
\hline
\end{tabular}

$\mathrm{NCl}$ CTC, National Cancer Institute Common Toxicity Criteria.
22 patients (71\%). Median TTP was 6.4 month, whereas median OS was 11.0 month. Tumour or response re-evaluation was performed every other cycle by the same radiologic imaging (CT or MRI). Patients needed to have stable disease for a minimum of 8 weeks to be considered 'disease control'.

Results are summarised in Table 3. Apart from the two patients mentioned above, there were no treatment-related deaths.

\section{DISCUSSION}

Unresectable BTC is associated with a poor prognosis and treatment options are limited. As palliative therapeutic approach in patients with unresectable BTC we assessed the combination chemotherapy of GEMOX-3. Our data demonstrate that in advanced BTC the GEMOX-3 protocol shows good antitumour activity and tolerable toxicity with 3 treatment days per month and administration in an outpatient setting.

In palliative intention, novel combination chemotherapies are being tested in order to increase RRs and survival without additional toxicity. Although the combination of GEM and CDDP showed favourable RRs (RR 36.6\%), the regimen was associated with a high frequency of grade 3 and 4 toxicities (Doval et al, 2004). At the start of the present study, two phase II trials have been published which analysed combinations with the new platinum component OX: GEM (fixed dose rate)/OX (RR 35.5\%, PFS 5.7 months, median OS 15.4 months) (Andre et al, 2004), and capecitabine/OX (RR 26.6\%, TTP NR, median OS NR) (Glover et al, 2005). Other combinations, for example, capecitabine/GEM, showed similar outcomes (RR 31\%, PFS 7 months, OS 14 months) (Knox et al, 2005) The results of our study (RR 26\%, TTP 6.4, OS 11.0) now underscore that GEMOX is an active combination chemotherapy with acceptable toxicity. The differences to the study of Andre et al (2004), might be explained by the inclusion of two groups of patients with different eligibility criteria in the French study (higher/lower bilirubin cutoff, better/worse performance status, previous chemotherapy). Although the group with stricter restrictions reached a RR of $35.5 \%$ and a superior OS of 15.4 months, the other group showed a RR of $22 \%$ and an inferior OS of 7.6 months. Longer infusion times of GEM (100 min vs $30 \mathrm{~min}$ ) and the application of OX on a second day by Andre et al (2004) might also have influenced the outcome. In general, considering the short OS of patients with advanced BTC inpatient chemotherapy protocols should be minimised.

Thrombocytopenia was the most frequent toxicity in our study, but had no major clinical impact. The frequent treatment delays (54\%) might be explained by the high thrombocyte cutoff level of $100 \times 10^{9} 1^{-1}$ chosen in the study and the cumulative toxicity of GEM and OX at the doses used. Another $9 \%$ of treatment delays were caused by infections of any kind, most frequently owing to cholangitis and occlusion of biliary stents in patients with

Table 3 Treatment efficacy $(n=31)$

\begin{tabular}{|c|c|c|c|c|c|c|c|c|}
\hline Treatment response & \multicolumn{2}{|c|}{ Overall } & \multicolumn{2}{|c|}{ Intrahepatic } & \multicolumn{2}{|c|}{ Gallbladder } & \multicolumn{2}{|c|}{ Extrahepatic } \\
\hline Complete response & 0 & 0 & 0 & 0 & 0 & 0 & 0 & 0 \\
\hline Partial response & 8 & $26(14-44)$ & I & 7 & 4 & 40 & 3 & 42 \\
\hline Stable disease & 14 & $45(29-62)$ & 9 & 64 & 3 & 30 & 2 & 29 \\
\hline Progressive disease & 9 & $29(16-47)$ & 4 & 29 & 3 & 30 & 2 & 29 \\
\hline Median OS (months) & & 11.0 & & & & & & \\
\hline
\end{tabular}

$\mathrm{Cl}$, confidence interval; OS, overall survival; TTP, time-to-progression. 
extrahepatic and perihilar tumours. Despite this fact, patients with intraductal growth type BTC, tended to have a better OS than patients with mass forming type BTC (OS 13.3 vs 8.4).

With respect to neurotoxicity there was a clear relation between cumulative OX dose and delayed sensory neuropathy, as described in the literature (Extra et al, 1998; de Gramont et al, 2000). After therapy discontinuation, however, there was a fast improvement of treatment-related symptoms.

The important limitation of our study is its single centre phase II design. Owing to the limited number of patients, it was not possible to perform a randomised study comparing GEMOX to a single agent. However, a disease control $>70 \%$ of patients suggests that patients with unresectable BTC benefit from the combination chemotherapy.

Some authors report an inferior RR in mass forming BTC (Nehls et al, 2003; Andre et al, 2004), whereas others document a more

\section{REFERENCES}

Agresti CB (1998) Approximate is better than 'Exact' for interval estimation of binomial proportions. Am Stat 52: 119-126

Alberts SR, Al-Khatib H, Mahoney MR, Burgart L, Cera PJ, Flynn PJ, Finch TR, Levitt R, Windschitl HE, Knost JA, Tschetter LK (2005) Gemcitabine, 5 -fluorouracil, and leucovorin in advanced biliary tract and gallbladder carcinoma: a North Central Cancer Treatment Group phase II trial. Cancer 103: $111-118$

Andre T, Tournigand C, Rosmorduc O, Provent S, Maindrault-Goebel F, Avenin D, Selle F, Paye F, Hannoun L, Houry S, Gayet B, Lotz JP, de Gramont A, Louvet C (2004) Gemcitabine combined with oxaliplatin (GEMOX) in advanced biliary tract adenocarcinoma: a GERCOR study. Ann Oncol 15: 1339-1343

Chang WH, Kortan P, Haber GB (1998) Outcome in patients with bifurcation tumors who undergo unilateral $v s$ bilateral hepatic duct drainage. Gastrointest Endosc 47: 354-362

de Gramont A, Figer A, Seymour M, Homerin M, Hmissi A, Cassidy J, Boni C, Cortes-Funes H, Cervantes A, Freyer G, Papamichael D, Le Bail N, Louvet C, Hendler D, de Braud F, Wilson C, Morvan F, Bonetti A (2000) Leucovorin and fluorouracil with or without oxaliplatin as first-line treatment in advanced colorectal cancer. J Clin Oncol 18: 2938-2947

de Groen PC, Gores GJ, LaRusso NF, Gunderson LL, Nagorney DM (1999) Biliary tract cancers. N Engl J Med 341: 1368-1378

Doval DC, Sekhon JS, Gupta SK, Fuloria J, Shukla VK, Gupta S, Awasthy BS (2004) A phase II study of gemcitabine and cisplatin in chemotherapy-naive, unresectable gall bladder cancer. $\mathrm{Br} J$ Cancer 90: $1516-1520$

Doval DC, Sekhon JS, Gupta SK, Gupta S, Awasthy BS (2002) Chemotherapy in biliary tract carcinomas: results in India. Semin Oncol 29: 46-50

Ducreux M, Van Cutsem E, Van Laethem JL, Gress TM, Jeziorski K, Rougier P, Wagener T, Anak O, Baron B, Nordlinger B (2005) A randomised phase II trial of weekly high-dose 5-fluorouracil with and without folinic acid and cisplatin in patients with advanced biliary tract carcinoma: results of the 40955 EORTC trial. Eur J Cancer 41: 398-403

Extra JM, Marty M, Brienza S, Misset JL (1998) Pharmacokinetics and safety profile of oxaliplatin. Semin Oncol 25: 13-22

Farley DR, Weaver AL, Nagorney DM (1995) Natural history. of unresected cholangiocarcinoma: patient outcome after noncurative intervention. Mayo Clin Proc 70: 425-429

Gallardo JO, Rubio B, Fodor M, Orlandi L, Yanez M, Gamargo C, Ahumada M (2001) A phase II study of gemcitabine in gallbladder carcinoma. Ann Oncol 12: $1403-1406$

Gebbia V, Giuliani F, Maiello E, Colucci G, Verderame F, Borsellino N, Mauceri G, Caruso M, Tirrito ML, Valdesi M (2001) Treatment of inoperable and/or metastatic biliary tree carcinomas with single-agent gemcitabine or in combination with levofolinic acid and infusional fluorouracil: results of a multicenter phase II study. J Clin Oncol 19: 4089-4091

Glover KY, Thomas MB, Brown TD, Hoff PM, Iwasaki M, Abbruzzese JL (2005) A phase II study of oxaliplatin and capecitabine (XELOX) in patients with unresectable cholangiocarcinoma, including carcinoma of the gallbladder and biliary tract. J Clin Oncol (Meeting Abstracts) 23: 338 (abstract 4123) aggressive biology of gallbladder cancer (Doval et al, 2004, Knox et al, 2005). Despite the data are limited by the low number of patients it should be noted that patients with gallbladder cancer and extrahepatic BTC showed a considerably better RR (40 and 42\%) than patients with intrahepatic BTC (RR 7\%). This interesting observation should be verified in a larger trial that allows for a differentiated subgroup analysis.

Furthermore, despite extensive diagnostic efforts it cannot be ruled out that also patients with a cancer of unknown primary site were included and lead to a bias in the data. More precise molecular profile of the three BTC growth types will be necessary to better distinguish BTC from other solid tumours.

In summary, GEMOX-3 is efficacious and can be safely given on an outpatient basis as a palliative chemotherapy of advanced BTC. These promising RRs now need to be verified in controlled phase III trials, as they are currently ongoing.
Khan SA, Taylor-Robinson SD, Toledano MB, Beck A, Elliott P, Thomas HC (2002) Changing international trends in mortality rates for liver, biliary and pancreatic tumours. J Hepatol 37: 806-813

Knox JJ, Hedley D, Oza A, Feld R, Siu LL, Chen E, Nematollahi M, Pond GR, Zhang J, Moore MJ (2005) Combining gemcitabine and capecitabine in patients with advanced biliary cancer: a phase II trial. J Clin Oncol 23: $2332-2338$

Kornek GV, Schuell B, Laengle F, Gruenberger T, Penz M, Karall K, Depisch D, Lang F, Scheithauer W (2004) Mitomycin C in combination with capecitabine or biweekly high-dose gemcitabine in patients with advanced biliary tract cancer: a randomised phase II trial. Ann Oncol 15: $478-483$

Kubicka S, Rudolph KL, Tietze MK, Lorenz M, Manns M (2001) Phase II study of systemic gemcitabine chemotherapy for advanced unresectable hepatobiliary carcinomas. Hepatogastroenterology 48: 783- 789

Kuhn R, Hribaschek A, Eichelmann K, Rudolph S, Fahlke J, Ridwelski K (2002) Outpatient therapy with gemcitabine and docetaxel for gallbladder, biliary, and cholangio-carcinomas. Invest New Drugs 20: $351-356$

Louvet C, Andre T, Lledo G, Hammel P, Bleiberg H, Bouleuc C, Gamelin E, Flesch M, Cvitkovic E, de Gramont A (2002) Gemcitabine combined with oxaliplatin in advanced pancreatic adenocarcinoma: final results of a GERCOR multicenter phase II study. J Clin Oncol 20: 1512-1518

Nakeeb A, Tran KQ, Black MJ, Erickson BA, Ritch PS, Quebbeman EJ, Wilson SD, Demeure MJ, Rilling WS, Dua KS, Pitt HA (2002) Improved survival in resected biliary malignancies. Surgery 132: 555-563; discussion 563-564

Nehls O, Oettle H, Hartmann J-T, Hofheinz R, Hochhaus A, Makowski M, Arnold D, Burkart C, Gregor M, Klump B (2003) Multicenter phase II trial of oxaliplatin plus capecitabine (XELOX) in advanced biliary system adenocarcinomas (study CCC/GBC-01). In Proc Am Soc Clin Oncol 22: 280 (abstract 1126)

Nehls O, Klump B, Arkenau HT, Hass HG, Greschniok A, Gregor M, Porschen R (2002) Oxaliplatin, fluorouracil and leucovorin for advanced biliary system adenocarcinomas: a prospective phase II trial. $\mathrm{Br} \mathrm{J}$ Cancer 87: $702-704$

Oertli D, Herzog U, Tondelli P (1993) Primary carcinoma of the gallbladder: operative experience during a 16 year period. Eur J Surg 159: 415-420

Patel T (2001) Increasing incidence and mortality of primary intrahepatic cholangiocarcinoma in the United States. Hepatology 33: 1353-1357

Patel $T$ (2002) Worldwide trends in mortality from biliary tract malignancies. BMC Cancer 2: 10

Patt YZ, Hassan MM, Aguayo A, Nooka AK, Lozano RD, Curley SA, Vauthey JN, Ellis LM, Schnirer II Wolff RA, Charnsangavej C, Brown TD (2004) Oral capecitabine for the treatment of hepatocellular carcinoma, cholangiocarcinoma, and gallbladder carcinoma. Cancer 101: 578-586

Patt YZ, Hassan MM, Lozano RD, Waugh KA, Hoque AM, Frome AI, Lahoti S, Ellis L, Vauthey JN, Curley SA, Schnirer II Raijman I (2001) Phase II trial of cisplatin, interferon alpha-2b, doxorubicin, and 5-fluorouracil for biliary tract cancer. Clin Cancer Res 7: 3375-3380

Penz M, Kornek GV, Raderer M, Ulrich-Pur H, Fiebiger W, Lenauer A, Depisch D, Krauss G, Schneeweiss B, Scheithauer W (2001) Phase II trial 
of two-weekly gemcitabine in patients with advanced biliary tract cancer. Ann Oncol 12: $183-186$

Raderer M, Hejna MHL, Valencak JB, Kornek GV, Weinländer GS, Bareck E, Lenauer J, Brodowicz T, Lang F, Scheithauer W (1999) Two consecutive phase II studies of 5-fluorouracil/leucovorin/mitomycin C and of gemcitabine in patients with advanced biliary cancer. Oncology 56: $177-180$

Simon R (1989) Optimal two-stage designs for phase II clinical trials. Control Clin Trials 10: $1-10$

Taieb J, Mitry E, Boige V, Artru P, Ezenfis J, Lecomte T, Clavero-Fabri MC, Vaillant JN, Rougier P, Ducreux M (2002) Optimization of 5-fluorouracil (5-FU)/cisplatin combination chemotherapy with a new schedule of leucovorin, 5-FU and cisplatin (LV5FU2-P regimen) in patients with biliary tract carcinoma. Ann Oncol 13: 1192-1196

Therasse P, Arbuck SG, Eisenhauer EA, Wanders J, Kaplan RS, Rubinstein L, Verweij J, Van Glabbeke M, van Oosterom AT, Christian MC, Gwyther SG (2000) New guidelines to evaluate the response to treatment in solid tumors. European Organization for Research and Treatment of Cancer, National Cancer Institute of the United States, National Cancer Institute of Canada. J Natl Cancer Inst 92: 205-216

Valencak J, Kornek GV, Raderer M, Ulrich-Pur H, Krauss G, Greul R, Pidlich J, Pfeffel F, Haider K, Kwasny W, Schneeweiss B, Scheithauer W (1999) Gemcitabine for the treatment of advanced biliary tract carcinomas: evaluation of two different dose regimens. Onkologie 22: 498-501 